\title{
Brachytherapy or enucleation in ring melanoma patients: which is better? Preliminary results of the authors' own experiences
}

\author{
Anna Markiewicz, MD, PhD 1.2, Karolina Gerba-Górecka, MD², Barbara Jakubowska, MD², \\ Magdalena Dębicka-Kumela, MD',2, Joanna Kowal, MD, PhD',2, Izabella Karska-Basta, MD, PhD',2 \\ Konrad Skórkiewicz, MSc ${ }^{2,3}$, Prof. Bożena Romanowska-Dixon, MD, PhD',2 \\ 'Department of Ophthalmology and Ocular Oncology, Faculty of Medicine, Jagiellonian University Medical College, Krakow, Poland, \\ ${ }^{2}$ Department of Ophthalmology and Ocular Oncology, University Hospital, Krakow, Poland, ${ }^{3}$ Jagiellonian University, Faculty of Physics, \\ Astronomy and Applied Computer Science, Krakow, Poland
}

\begin{abstract}
Purpose: A retrospective evaluation of effectiveness of brachytherapy or enucleation in treatment of rare form of uveal melanoma: ring melanoma.

Material and methods: Analysis comprised 49 patients treated from 2000 to 2019 for ring melanoma involving ciliary body. In 15 patients, primary treatment consisted of brachytherapy $\left({ }^{106} \mathrm{Ru}\right.$ or $\left.{ }^{125} \mathrm{I}\right)$, whereas in 34 patients, eyeballs were enucleated. The evaluation concerned differences between analyzed groups relating to the clinical and histopathological features as well as overall survival, cancer-specific overall survival, and disease-free survival.

Results: No significant differences between the analyzed groups were found with regards to clinical and histopathological features, apart from intra-ocular pressure (increased in the enucleation group). Kaplan-Meier analysis did not reveal any significant differences between the group treated with enucleation and the group undergoing brachytherapy, both with regards to overall survival $(p=0.325)$ and cancer-specific overall survival $(p=0.477)$. A significant difference was observed in disease-free survival $(p=0.009)$, which was significantly shorter in the group undergoing brachytherapy. In the analysis of parameters of the applied brachytherapy, no significant differences between patients with and without local recurrence were found. Mean observation period was 350.8 weeks (range, 24-996 weeks, SD = 231.6). A local recurrence occurred in $11(22.4 \%)$ patients, including $3(6.1 \%)$ in enucleation and $8(16.3 \%)$ after brachytherapy groups. Metastasis developed in $11(22.4 \%)$ cases after a mean follow-up of 133 weeks (33.25 months), range 3-655 weeks.

Conclusions: Preliminary observations may suggest that brachytherapy in this rare form of uveal melanoma, such as ring melanoma involving the ciliary body, may be taken into consideration as a useful alternative to enucleation. However, the confirmation of such an approach requires a larger patients' group to be gathered, and also a longer follow-up period. This is especially important in patients with a good baseline visual acuity in the affected eye, or when
\end{abstract} the neoplasm is present in the remaining eye with vision.

J Contemp Brachytherapy 2021; 13, 4: 433-440 DOI: https://doi.org/10.5114/jcb.2021.108598

Key words: ring melanoma, brachytherapy, enucleation, ocular melanoma, uveal melanoma, melanoma.

\section{Purpose}

Ring melanoma is a rare form of the most frequent primary inter-ocular neoplasm in adults, i.e., uveal melanoma, and has a poorer prognosis than all other types of neoplasms [1-4].

Published data suggest that iris melanoma and ciliary body melanoma make up $2-4 \%$ and $4-10 \%$ of all uveal melanomas, out of which only $10 \%$ constitute ring melanomas. In other words, ring melanoma accounts for
$0.3-0.4 \%$ of all uveal melanomas [5, 6]. Assuming that the prevalence of uveal melanoma is 1.3-8.6 of new cases per 1,000,000 people, it may be estimated that for ring melanoma, there will be $0.78-12$ new cases per year per $100,000,000$ individuals.

Uveal ring melanoma is defined in many ways. According to Reese, this term may signify 'disseminated' ring melanoma, which involves the iris or iridocorneal angle, and 'true' ring melanoma involving the ciliary body [8]. At a later stage, the 'disseminated' ring melano-
Address for correspondence: Anna Markiewicz, MD, PhD, Department of Ophthalmology and Ocular Oncology, Faculty of Medicine, Jagiellonian University Medical College, 38 Kopernika St., 31-501 Krakow, Poland, phone: +48 12-424-75-40, fax: +48 12-424-75-63,

๑e-mail: anna.markiewicz@uj.edu.pl or annamarkiewicz@interia.pl
Received: 03.01.2021

Accepted: 16.04 .2021

Published: 24.08 .2021 
ma is associated with a diffuse iris melanoma (DIM) and iris ring melanoma; and this classification is now universally used [2, 4]. Generally, it is assumed that ring melanoma is a uveal melanoma, which in a circumferential manner (360 degrees) involves the eyeball. However, its variations, in which the tumor involves the iris or ciliary body on the area exceeding 6 clock hours, or in the case when there are numerous foci of the pigment. or when the iridocorneal angle is simply 'covered' with a dark pigment looking suspiciously on the area exceeding 180 degrees, are also classified as ring melanomas [1-3, 9-11].

At times, it is difficult to determine definitively the point of origin of this type of neoplasm. Ring melanoma may involve the iris, iridocorneal angle, and the ciliary body in a way that each of these structures is affected separately. However, more frequent forms involve various combinations, in which the melanoma occurs simultaneously in two or even three of the above places, but the ciliary body is the main site of involvement [10, 12]. There are also reports presenting isolated cases of uveal ring melanoma [9].

Clinical manifestations of ring melanoma may vary, depending on its location. A progressing change of the iris color in one eye (heterochromia) that has not occurred since birth, with an increased pigmentation over the passage of time, which may be accompanied by an elevated intra-ocular pressure in one eye, may be indicative of iris ring melanoma. When the cancer involves the iridocorneal angle, intra-ocular pressure increases as melanoma cells or tumor pigment-laden macrophages block the trabecular meshwork and Schlemm's canals, inhibiting the outflow of aqueous humour from the eyeball. The growth of ring melanoma within the ciliary body can also lead to secondary glaucoma; additionally, there are dilated episcleral vessels, such as tumor sentinel vessels, and also the foci of extraocular infiltration.

Clinical symptoms involving visual disturbances occur relatively late, and the majority of patients have a very good acuity of vision at the moment of diagnosis [1-3, 11, 12]. Generally, atypical clinical picture of the condition and rarity of incidence result in a delayed diagnosis. This, in turn, leads to a delay in initiating the correct treatment, as opposed to other types of uveal melanomas, which are usually well-circumscribed tumors [1, 11, 12]. Furthermore, difficulties with incorrect diagnosis in ring melanoma lead to the use of various procedures, including surgical ones, which increase the risk of systemic disseminated neoplasm. Most frequently, this situation occurs when anti-glaucoma filtration procedures are administered for incorrectly diagnosed pigmentary glaucoma, or with cyclo-destruction of the ciliary body or cataract removal. All the above procedures increase the risk of mortality in patients with ring melanoma $[4,11,13,14]$.

Practically, the overwhelming majority of ring melanoma cases within this study were treated with the eyeball enucleation. Only in a few cases, brachytherapy or proton therapy were applied [1-3, 15].

For most patients, the diagnosis of a malignant tumor, including ring melanoma, and the recommendation of eyeball enucleation as the sole treatment method, is a terrible shock. Decision to start the treatment is very stressful, es- pecially when the patient has full visual acuity in the affected eye. That is why some patients refrain from deciding to remove the eyeball, or even give up or delay the treatment. Only severe pain forces patients to start the therapy.

For the above reasons, brachytherapy was used in patients who did not decide to undergo enucleation or in those who had only one eye, the only eye with vision, in which ring melanoma was present. In some cases, brachytherapy was performed to obtain a histopathological specimen confirming the presence of intra-ocular tumors and to prove its existence to the patient.

The objective of this work was to compare the results of ring melanoma treatment in our center, involving either enucleation or brachytherapy. Also, according to our clinical experience, this paper aimed to answer the question in the title: In uveal/ocular ring melanoma patients, which treatment is more effective - brachytherapy or enucleation?

\section{Material and methods}

\section{Inclusion and exclusion criteria}

In our center, from 2000 to 2019, ring melanoma was diagnosed in 62 patients. With regards to better prognosis of ring melanoma of the iris, without involvement of ciliary body, 8 cases of diffuse iris melanoma were excluded from the study, and they will be the subject of a separate analysis. In one patient, ring melanoma with uveal involvement up to the area of the optic disc was diagnosed; this case was also not taken into further consideration. Additionally, 4 more patients were excluded from the study, two of them did not turn up for the planned treatment, one more patient did not appear for any follow-up visit after the enucleation, and one patient decided to undergo proton therapy in another center. Finally, 49 patients treated for true ring melanoma with the ciliary body involvement, treated either with ${ }^{106} \mathrm{Ru}$ or ${ }^{125}$ I brachytherapy, or those who underwent enucleation were included in the study.

The diagnosis of ring melanoma was made on the basis of ophthalmological assessment covering the anterior segment of the eye in the slit lamp, gonioscopy, transillumination (blockage of the light in the ciliary body at the tumor site was observed in $100 \%$ patients), eye fundus examination, with dilation of the pupil, anterior segment ultrasound (ultrabiomicroscopy - UBM), or on the basis of a histopathological assessment. Wherever possible, a photo documentation was made. In the analyzed patients' group, true ring melanoma was diagnosed when in clinical or histopathological picture, the neoplasm involved not less than 180 degrees, i.e., 6 clock hours of the ciliary body with a possible involvement of the iridocorneal angle or the iris. Figure 1 presents one of our patient's examination results.

\section{Parameters analyzed}

The study considered a retrospective analysis of the data collected in the files of patients, in whom true ring melanoma was diagnosed. The survival was evaluated comparing overall survival (OS), cancer-specific overall 

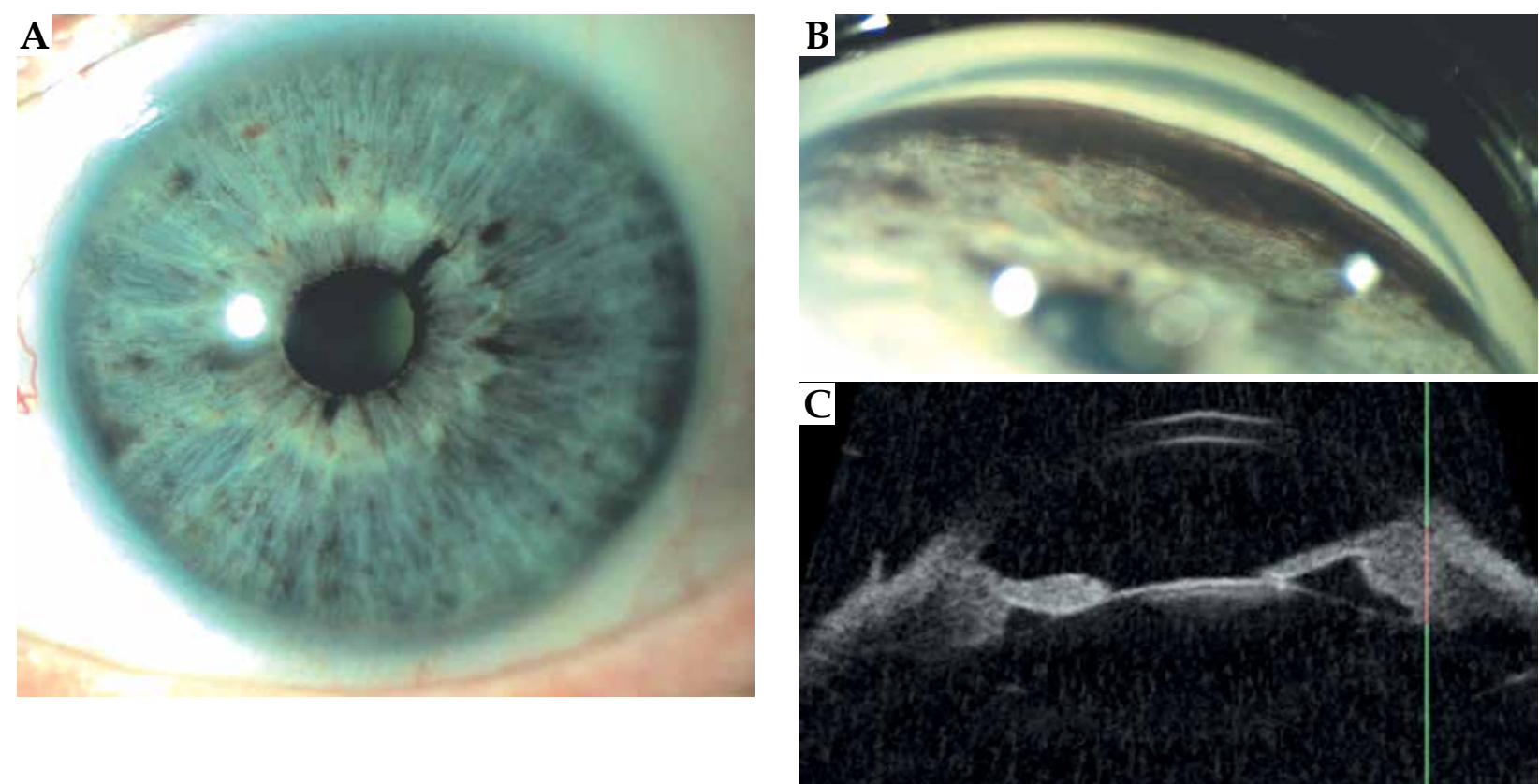

Fig. 1. A case with ring melanoma. A) An anterior segment examination: the color of peripheral part of the iris is darker. B) Gonioscopy: the dark brown tumor involved all trabecular meshwork. C) Ultrabiomicroscopy (UBM) - transversal scan: the tumor involved all ciliary body

survival (CSOS), disease-free survival (DFS), and the risk of developing distant metastases, depending on the applied treatment method. The question that was pursued was whether there were any differences in clinical and histopathological pictures between the group treated with brachytherapy and the group where enucleation was performed, which could affect the survival results. Additionally, brachytherapy parameters, such as the dose per tumor apex, sclera, lens, and application time span were recorded.

\section{Qualification for treatment and its course}

Suturing a plaque with a radioactive isotope was performed in an operating theatre, in the majority of cases under local anesthesia and sedo-analgesia. Ruthenium-106 (CCB) applicators with a diameter of $20 \mathrm{~mm}$ (when tumor thickness was smaller than $5 \mathrm{~mm}$ ), and 14-seeds ${ }^{125} \mathrm{I}$ plaque with a diameter of $20.5 \mathrm{~mm}$ (when tumor thickness was larger than $5 \mathrm{~mm}$ ) were used for the therapy. A round applicator with a diameter of 20 or $20.5 \mathrm{~mm}$ was placed on the entire surface of the cornea, after previous application of protective contact lens, so that the applicator could cover the entire surface of the cornea and, symmetrically, the structures around the corneal limbus. Since the cornea's diameter, on average, measures $11.5 \mathrm{~mm}$, it was possible to irradiate the region of about $4.5 \mathrm{~mm}$ around corneal limbus, including the area of the ciliary body. The plaque was sutured temporarily with intra-scleral sutures for immobilization. The parameter, which was always taken into consideration was the total dose prescription point (tumor apex dose). In the case of a flat tumor infiltration, the thickness was assumed as $2 \mathrm{~mm}$, and when the tumor thickness was larger $2 \mathrm{~mm}$, the largest measurement was considered.
For every patient, an irradiation plan was individually prepared using Plaque Simulator $X$, version 5.3.9, so that total dose prescription point could vary between 80 and $120 \mathrm{~Gy}$, with the treatment duration not shorter than 43 and not longer than 168 hours.

Iodine-125 and ${ }^{106} \mathrm{Ru}$ brachytherapy of intra-ocular tumors was carried out in accordance with the guidelines and the procedures adopted at the University Hospital, which are compliant with the legal standards binding in our country.

Enucleation was performed in a typical manner with the eye movement muscle reconstruction, suturing the deep tissues and conjunctiva, and, from 2008 onwards, with the implantation of the orbital Guthoff implant in the absence of the extraocular infiltration. In some patients, in order to confirm the diagnosis histopathologically and also before the final decision concerning the type of treatment, the lesion was biopsied. Brachytherapy was applied in those patients who refused enucleation or in those where the neoplasm was present in the only eye with vision.

\section{Statistical analysis}

Distributions of qualitative variables were presented as counts $(n)$ and percentages (\%), using mean and standard deviation (SD). Relationships between two categorical variables were estimated with Fisher's exact test for $2 \times 2$ tables, and with Fisher-Freeman-Halton test for other tables. A comparison of distributions of quantitative variables between two independent groups was performed using Mann-Whitney test. Relationships between applied treatment and survival time was analyzed with Kaplan-Meier method and presented as survival functions. Significance of the differences between the an- 
alyzed survival functions were assessed using log-rank test. Differences with $p$-value less than 0.05 were treated as statistically significant. Statistical analyses were performed using IBM SPSS Statistics 26 for Windows (IBM Corp., released 2017, IBM SPSS Statistics for Windows, version 26.0; Armonk, NY, IBM Corp., USA).

The Authors declare that this investigation was carried out following the rules of the Declaration of Helsinki of 1975 (revised in 2013).

\section{Results}

The analyzed group consisted of 25 (51.0\%) women and $24(49.0 \%)$ men, and the mean age was 58.5 years (range, 19-79 years, SD $=14.8$ ), whilst the mean observation period was 350.8 weeks (range, 24-996 weeks,
$\mathrm{SD}=231.6$ ). Enucleation was performed in 34 patients $(69.4 \%)$, whereas brachytherapy in 15 patients $(30.6 \%)$, including 2 cases with iodine brachytherapy $\left({ }^{125} \mathrm{I}\right)$ and 13 with ruthenium $\left({ }^{106} \mathrm{Ru}\right)$.

The TNM classification of the disease stage indicated that $45(91.8 \%)$ tumors were $\mathrm{T} 4 \mathrm{~b}$ and $4(8.2 \%)$ were $\mathrm{T} 4 \mathrm{~d}$, which corresponded to stage IIIB and IIIC of the $8^{\text {th }}$ edition staging for uveal melanoma. There were no statistically significant differences between the groups with regards to the cancer stage [16]. Extraocular infiltration at the moment of diagnosis was observed in $3(6.1 \%)$ patients treated with enucleation and in $1(2.0 \%)$ patient treated with brachytherapy. The only feature in the clinical picture, which significantly differed one group from another was increased intra-ocular pressure (above $21 \mathrm{mmHg}$ ). Its values in the patients undergoing enu-

Table 1. Clinical and histopathological characteristics of the analyzed group of ring melanoma patients, divided into the type of treatment applied

\begin{tabular}{|c|c|c|c|c|}
\hline Parameter & Enucleation, $n(\%)$ & Brachytherapy, $n$ (\%) & Total, $n(\%)$ & $p$-value \\
\hline Sex & & & & 0.538 \\
\hline Female & $16(47.1)$ & $9(60.0)$ & $25(51.0)$ & \\
\hline Male & $18(52.9)$ & $6(40.0)$ & $24(49.0)$ & \\
\hline Affected eye & & & & 0.210 \\
\hline Left & $11(32.4)$ & $8(53.3)$ & $19(38.8)$ & \\
\hline Right & $23(67.6)$ & $7(46.7)$ & $30(61.2)$ & \\
\hline Visual acuity upon diagnosis & & & & 0.362 \\
\hline$<0.1$ & $9(26.5)$ & $1(6.7)$ & $10(20.4)$ & \\
\hline $0.1-0.2$ & $3(8.8)$ & $3(20.0)$ & $6(12.2)$ & \\
\hline $0.3-0.6$ & $5(14.7)$ & $3(20.0)$ & $8(16.3)$ & \\
\hline $0.7-1.0$ & $17(50.0)$ & $8(53.3)$ & $25(51.0)$ & \\
\hline Intra-ocular pressure & & & & 0.017 \\
\hline Correct & $6(17.6)$ & $8(53.3)$ & $14(28.6)$ & \\
\hline Hight $>21 \mathrm{~mm} \mathrm{Hg}$ & $28(82.4)$ & $7(46.7)$ & $35(71.4)$ & \\
\hline Clinical assessment & & & & 0.319 \\
\hline \multicolumn{5}{|c|}{ Tumor involvement in clock hours } \\
\hline $6-8$ & $8(23.5)$ & $7(46.7)$ & $15(30.6)$ & \\
\hline $9-11$ & $5(14.7)$ & $2(13.3)$ & $7(14.3)$ & \\
\hline 12 & $21(61.8)$ & $6(40.0)$ & $27(55.1)$ & \\
\hline Gonioscopy & & & & 0.061 \\
\hline \multicolumn{5}{|c|}{ Tumor involvement in clock hours } \\
\hline $6-8$ & $5(14.7)$ & $3(23.1)$ & $8(17.0)$ & \\
\hline $9-11$ & $4(11.8)$ & $5(38.5)$ & $9(19.1)$ & \\
\hline 12 & $25(73.3)$ & $5(38.5)$ & $30(63.8)$ & \\
\hline Pigmentation & & & & 0.089 \\
\hline Heavily & $34(100.0)$ & $13(86.7)$ & $47(95.9)$ & \\
\hline Moderate & $0(0.0)$ & $2(13.3)$ & $2(4.1)$ & \\
\hline Amelanotic & $0(0.0)$ & $0(0.0)$ & $0(0.0)$ & \\
\hline Iris color & & & & 0.434 \\
\hline Blue & $26(76.5)$ & $14(93.3)$ & $40(81.6)$ & \\
\hline Green & $5(14.7)$ & $1(6.7)$ & $6(12.3)$ & \\
\hline Brown & $3(8.8)$ & $0(0.0)$ & $3(6.1)$ & \\
\hline Total & $34(100.0)$ & 15 (100.0) & 49 (100.0) & \\
\hline
\end{tabular}


cleation were significantly higher $(p=0.017)$. Other clinical data of the study groups are presented in Table 1. In $41(83.7 \%)$ cases, in which a histopathological assessment was performed, a mixed type of melanoma dominated; it was diagnosed in 29 (59.2\%) patients after enucleation and in the specimen harvested before brachytherapy in $5(10.2 \%)$ cases. An epithelioid cell type was observed in $5(10.2 \%)$ patients and $1(2.0 \%)$ case, respectively. Only $1(2.0 \%)$ patient undergoing brachytherapy had the spindle-cell type of uveal melanoma. No significant differences in the histopathological type of melanoma was observed between the groups undergoing various treatment methods $(p=0.581)$. In a significant majority of patients, i.e. in $46(93.9 \%)$, the iris of the affected eye was of a light color (blue or green).

\section{Recurrence and subsequent treatment}

A local recurrence of the neoplasm occurred in a total of $11(22.4 \%)$ patients; in $3(6.1 \%)$ patients with enucleation, a recurrence had the form of nodules of the orbit. All the patients were treated with the excision of the lesions with a healthy tissue margin. No case of second recurrence was observed. In the patients after brachytherapy, a recurrence was observed in $8(16.3 \%)$ cases. The recurrence was defined as an increase in the tumor size in UBM and a clinical increase of the area affected by melanoma (on the basis of comparison of photo documentation). In $2(4.1 \%)$ patients, the eyeball was removed and in $6(12.2 \%)$ cases, next brachytherapy was performed, as the patients did not agree for enucleation. In $2(4.1 \%)$ patients after the second brachytherapy, enucleation was still performed due to second relapse. In the second group of patients where the primary enucleation was performed, the frequency of local recurrence was significantly lower than in the group, in which the patients were primarily treated with brachytherapy $(p=0.001)$.

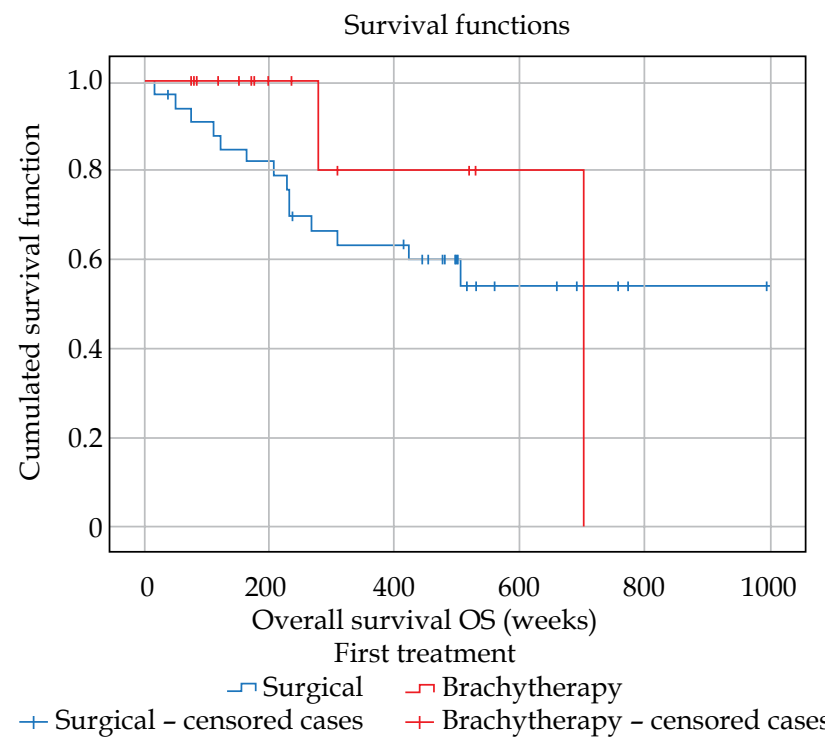

Fig. 2. Overall survival (in weeks) in true ring melanoma patients for the group treated with enucleation (blue line) and the group treated with brachytherapy (red line). Kaplan-Meier analysis, $p=0.325$

\section{Metastases}

The studied groups did not differ significantly from each other with respect to the number of people, in whom distant metastases were found $(p=0.464)$. Cancer metastases (in the majority of cases to the liver, $100 \%$ ) occurred in $9(18.4 \%)$ patients treated with enucleation, and in $2(4.1 \%)$ treated with brachytherapy. Metastasis developed after a mean follow-up period of 133 weeks (33.25 months), ranging 3-655 weeks.

\section{Survival}

Kaplan-Meier analysis did not reveal any significant differences between the patient's group treated with eyeball removal and the group undergoing brachytherapy for ring melanoma, both with regards to OS $(p=0.325)$ and CSOS $(p=0.477)$, which is illustrated in Figures 2 and 3. The evaluation of DFS $(p=0.009)$ showed one significant difference between the groups (Figure 4): in the patients treated with brachytherapy, a neoplasm recurrence was significantly more frequent. Also, the correlations between the 'tumor involvement in clock hour' in clinical picture and in gonioscopy, and DFS were analyzed $(p=0.706$ and $p=0.949$, respectively), OS ( $p=0.906$ and $p=0.602$, respectively), and $\operatorname{CSOS}(p=0.855$ and $p=0.888$, respectively), with no statistical significance obtained.

\section{Brachytherapy parameters}

Table 2 presents the parameters of brachytherapy of the ring melanoma patients treated with primary therapy (the first treatment) and secondary therapy (the second treatment) due to a relapse. The analysis did not show any significant differences in brachytherapy parameters, such as irradiation time $(p=0.925)$, total dose prescription point $(p=0.710)$, average dose-rate prescription point $(p=0.620)$, average dose-rate sclera $(p=0.534)$, and to-

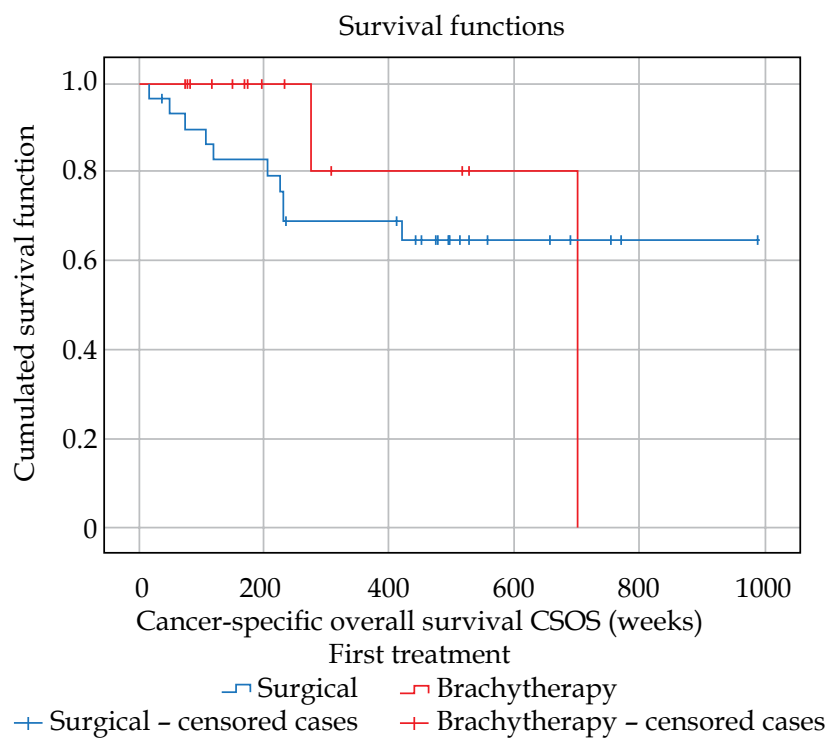

Fig. 3. Cancer-specific overall survival (in weeks) in true ring melanoma patients for the group treated with enucleation (blue line) and the group treated with brachytherapy (red line). Kaplan-Meier analysis, $p=0.477$ 


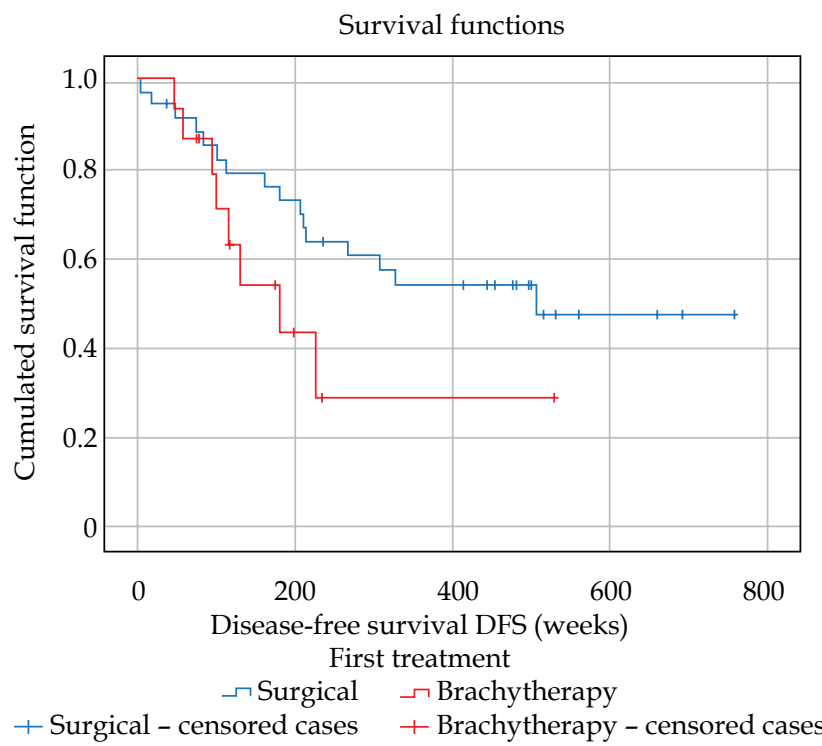

Fig. 4. Disease-free survival (in weeks) in true ring melanoma patients for the group treated with enucleation (blue line) and for the group treated with brachytherapy (red line). Kaplan-Meier analysis, $p=0.009$

tal dose sclera $(p=0.181)$ between the two groups of patients, including those undergoing brachytherapy, in whom a local recurrence was observed, and those after brachytherapy and without a relapse.

\section{Discussion}

Ring melanoma is a rare form of the most frequent primary malignant intra-ocular tumor in adults, i.e. uveal melanoma, and it is characterized by a poor prognosis. This is influenced by a delayed diagnosis, often preceded by a misdiagnosis with therapeutic procedures, and frequent surgical procedures, which increase the risk of systemic dissemination of the neoplasm through the lymphatic vessels [4, 11]. Additionally, melanoma involvement of a larger area (at least 6 clock hours) of the anterior segment of uvea, including richly vascularized ciliary body has a detrimental effect on the prognosis [5]. Regarding large extensiveness of the eye tissues involvement with ring melanoma, eyeball enucleation is a generally accepted treatment method, especially when the neoplasm also affects the ciliary body, which represent a case of 'true ring melanoma' [1]. In a situation where ring melanoma involves only the iris, more and more centers currently select conservative treatment with radiotherapy, proton therapy, or brachytherapy using individually prepared applicators with radioactive isotopes, such as ${ }^{106} \mathrm{Ru}$ or ${ }^{125} \mathrm{I}$ [17-22]. In the case of ring melanoma with ciliary body involvement, enucleation still remains the sole treatment method [1].

In the analyzed group of patients with ring melanoma of the ciliary body, $51 \%$ of cases had very good visual acuity (best corrected visual acuity $[\mathrm{BCVA}]=0.7-1.0$ ). In such a situation, the decision to remove the eyeball is very hard to make for the patient. A solution may be to obtain a specimen or fine needle aspiration biopsy, which proves to the patient that they are suffering from a malignant melanoma of the eyeball despite the lack or a low burden of existing symptoms [4].

In our department, within the last 20 years of clinical practice, we have seen $15(30.6 \%)$ patients who refused treatment of true ring melanoma with enucleation, and also patients with only one eye capable of vision, in which the cancer was present. The case of brachytherapy application in the diffuse melanoma of iris and ciliary body with the presence of extraocular nodular infiltrations with very good therapeutic effects was described by Gray et al. [23]. With the lack of consent for the removal of the eyeball, a decision to treat the patients with brachytherapy was undertaken.

Given the above situation, we have decided to analyze the results of the treatment with brachytherapy or enucleation in the patients with diagnosed ring melanoma with ciliary body involvement.

In the present study, the analyzed groups of patients did not differ significantly with regards to clinical features (except for the increased intra-ocular pressure), histopathological features, and neoplasm stages. This allows us to believe that the obtained results concerning the survival in both the groups are credible. Both OS and CSOS did not vary much in both the groups. That is why it may be assumed that the long-term results of treatment of patients with a diagnosis of true ring melanoma do not depend on a therapeutic method used. It is irrespective of whether it is brachytherapy or eyeball enucleation, the prognosis will be similar. In our opinion, this is very important observation allowing for a free choice of treatment method in this sub-type of uveal melanoma. It is especially important, as up till now, a great majority of ring melanoma have been treated with enucleation, a procedure, which many patients regard as very mutilating $[1,11,12,15]$.

Table 2. Selected brachytherapy parameters applied during primary (I) and secondary (II) treatments, and in the case of local recurrence. The mean value of the dose applied is presented in brackets

\begin{tabular}{lccc} 
Parameter & I treatment ${ }^{106} \mathrm{Ru}$ & I treatment ${ }^{125}$ I & II treatment ${ }^{106} \mathrm{Ru}$ \\
$n=13$ & $48.0-93.5(91.2)$ & $47.8-48(47.9)$ & $49.1-144.2(93.9)$ \\
\hline Irradiation time (h) & $105.5-153.8(108.5)$ & $106.7-182.7(144.7)$ & $61.9-91.75(68.3)$ \\
\hline Avg. dose-rate prescription point (cGy/h) & $83.12-110.9(98.3)$ & $87.4-96.7(92.0)$ & $102.4-106.9(103.9)$ \\
\hline Total dose prescription point (Gy) & $166.7-420.2(206.6)$ & $442.8^{*}$ & $178.7-185.4(183.4)$ \\
\hline Total dose sclera (Gy) & $23.9-95.0(50.5)$ & $95.1^{*}$ & $48.0-63.2(54.4)$
\end{tabular}

* No doses of total dose sclera and total dose lens for the second patient 
Additionally, as reported by other authors, a large rate of patients with true ring melanoma have a good visual acuity at baseline. This means that in these patients, the use of brachytherapy as a primary treatment would be much better, since this method allows for eyeball preservation and, for some time, a useful acuity of vision [1, 11].

Moreover, if in the majority of cases, the local treatment or anti-glaucoma procedures (only after brachytherapy) allows for normalizing inter-ocular pressure, the use of brachytherapy to preserve visual acuity and the eyeball in patients with true ring melanoma would be justified. It is generally believed that after radiotherapy, some procedures can be performed, such as cataract removal, antiglaucoma surgeries, and also anti-VEGF and triamcinolone injections into the anterior chamber or intravitreal, as a treatment of post-irradiation complications $[5,24]$.

Enucleation is always the final option. Some patients, as it was mentioned here before, in spite of the presence of a malignant tumor, do not consent for enucleation. Our observations confirm that it is easier for a patient to decide to have the eyeball removed only after the failure of the first treatment and sometimes, after the failure of the second. A relapse after brachytherapy treatment was found in $8(53.3 \%)$ patients; however, in $4(26.6 \%)$ of them, after the second brachytherapy, a positive result of local treatment was obtained and it persisted for the entire follow-up period. To summarize, in 11 (73.3\%) patients treated with brachytherapy, a good local final treatment result was achieved. In the published data, the frequency of local recurrence in the case of melanoma of the anterior part of uvea, including the ciliary body, is $0-55 \%$, and in our case, it was $26.7 \%[25,26]$. Despite the local recurrence, the 5 -year mortality in the group of our patients undergoing brachytherapy was $20.0 \%$, which is a result similar to those reported by other authors, who defined it to be $19.0 \%$ [27].

In the analyzed group of true ring melanoma patients, there was one significant difference observed in the groups undergoing various types of therapy with regards to DFS. Disease-free survival was significantly shorter in the group of patients treated with brachytherapy. This fact is associated with frequent local recurrences in the group undergoing radiotherapy. Fortunately, this fact did not lead to a poorer prognosis, which has already been mentioned here.

We also evaluated whether the applied brachytherapy parameters differed between the group without the relapse and the group with the local recurrence. The analysis, which was carried out did not reveal any significant differences in brachytherapy parameters, such as irradiation time $(p=0.925)$, total dose prescription point $(p=0.710)$, average dose-rate prescription point $(p=0.620)$, average dose-rate sclera $(p=0.534)$, and total dose sclera ( $p=0.181$ ) between the groups.

A good solution in the radiotherapy treatment of true ring melanoma may be the use of individually prepared applicators or the use of proton therapy, as it is done in unresectable cases or in diffuse melanomas, such as iris melanoma. The choice of such methods of treatment, along with the option of sparing stem cells of the limbus to avoid various complications, seem to be a rational solution in the conservative treatment of true ring melanoma [17-20].

Messer et al. analyzed 7,096 uveal melanoma patients with regards to survival depending on the fact whether the patient was treated with brachytherapy or enucleation. On the basis of their investigation performed, they concluded that tumor genetics, size, and early micro-metastatic spread were the most important indicators for survival. Therefore, local treatments other than enucleation, such as eye plaque brachytherapy, should be safe and efficient in patient's vision and aesthetics preservation. Their findings suggest that eye plaque brachytherapy is a reasonable treatment option for all size ocular melanoma tumors, even large size tumors [28]. The above observation may also confirm our results, such as the lack of differences in OS and CSOS, irrespectively of the ring melanoma treatment manner.

The group of patients with true ring melanomas analyzed in our study was one of the largest group described in English language publications. According to our best of knowledge, this is also the first publication evaluating the differences in the treatment of this condition with two diverse methods: conservative (in the form of brachytherapy) and radical (eyeball enucleation). We have shown that the choice of the former method of treatment dis not affect the length of survival of patients with ring melanoma involving the ciliary body.

Certainly, a prolongation of the follow-up period would be advisable as well as analyses performed on a larger group of patients with true ring melanoma. Due to very low prevalence of ring melanoma involving the ciliary body, a good idea would be to create a multi-center study, in which the center dealing with ophthalmological oncology could participate. This would allow to gather an adequately large patients' group for further analyses.

\section{Conclusions}

Preliminary observations may suggest that brachytherapy in this rare form of uveal melanoma, such as ring melanoma involving the ciliary body, may be taken into consideration as a useful alternative to enucleation. However, the confirmation of such an approach would require a larger patients' group to be investigated and a longer follow-up period. This is especially important in patients with good visual acuity in the affected eye or in cases where the cancer is present in the only eye with vision.

\section{Disclosure}

The authors report no conflict of interest.

\section{References}

1. Demirci H, Shields CL, Shields JA. Ring melanoma of the ciliary body: report on twenty-three patients. Retina 2002; 22: 698-706.

2. Demirci H, Shields CL, Shields JA. Diffuse iris melanoma: a report of 25 cases. Ophthalmology 2002; 109: 1553-1560.

3. Demirci H, Shields CL, Shields JA. Ring melanoma of the anterior chamber angle: a report of fourteen cases. Am J Ophthalmol 2001; 132: 336-342. 
4. Char DH, Kemlitz AE, Miller T. Iris ring melanoma: fine needle biopsy. Br J Ophthalmol 2006; 90: 420-422.

5. Kaliki S, Shields CL. Uveal melanoma: relatively rare but deadly cancer. Eye 2017; 31: 241-257.

6. Singh AD, Turell ME, Topham AK. Uveal melanoma: Trends in incidence, treatment, and survival. Ophthalmology 2011; 118: 1881-1885.

7. Virgili G, Gatta G, Ciccolallo L. Incidence of uveal melanoma in Europe. Arch Ophthalmol 2007; 114: 2309-2315.

8. Reese AB. Tumours of the eye. Cassel, London 1951.

9. Fitzpatrick M, Augsburger JJ, Koreishi FM. Complete ring melanoma of the choroid. Retina 1996; 16: 228-231.

10. Manschot WA. Ring melanoma. Arch Ophthalmol 1964; 71: 625-632.

11. Lee V, Cree IA, Hungerford JL. Ring melanoma - a rare cause of refractory glaucoma. Br J Ophthalmol 1999; 83: 194-198.

12. Omulecki W, Pruszczynski M, Borowski J. Ring melanoma of the iris and ciliary body. Br J Ophthalmol 1988; 69: 514-518.

13. Ardjomand N, Komericki P, Langmann G. Lymph node metastases arising from uveal melanoma. Wien Klin Wochenschr 2005; 117: 433-435.

14. Singer PR, Krupin T, Morton ES. Recurrent orbital and metastatic melanoma in patient undergoing previous glaucoma surgery. Am J Ophthalmol 1979; 87: 766-768.

15. Demirci H, Finger PT, Cocker R. Unusual presentation of diffuse melanoma of the iris. Br J Ophthalmol 2000; 84: 1076-1078.

16. Gershenwald JE, Scolyer RA. Melanoma staging: American Joint Committee on Cancer (AJCC) 8th edition and beyond. Ann Surg Oncol 2018; 25: 2105-2110.

17. Shields CL, Naseripour M, Shields JA. Custom-designed plaque radiotherapy for nonresectable iris melanoma in 38 patients: tumor control and ocular complications. Am J Ophthalmol 2003; 135: 648-656.

18. Scanderbeg DJ, Vasudev D, Rice RK. A modified COMS plaque for iris melanoma. J Contemp Brachytherapy 2011; 3: 131-133.

19. Shields CL, Shields JA, De Potter P. Treatment of non-resectable malignant iris tumours with custom designed plaque radiotherapy. Br J Ophthalmol 1995; 79: 306-312.

20. Leblanc A, Lumbroso-Le Rouic L, Desjardins L. Diffuse iris melanoma: conservative treatment with proton beam therapy after limbal stem cell preservation or enucleation? Ocul Oncol Pathol 2019; 5: 396-401.

21. Shields CL, Shah SU, Bianciotto CG. Iris melanoma management with iodine- 125 plaque radiotherapy in 144 patients: impact of melanoma-related glaucoma on outcomes. Ophthalmology 2013; 120: 55-61.

22. Willerding GD, Cordini D, Hackl C. Proton beam radiotherapy of diffuse iris melanoma in 54 patients. $\mathrm{Br}$ J Ophthalmol 2015; 99: 812-816.

23. Gray ME, Corrêa ZM, Augsburger JJ. Ciliary body melanoma with limited nodular extrascleral extension and diffuse iris-angle infiltration treated by whole anterior segment plaque radiotherapy. Int Ophthalmol 2007; 27: 273-276.

24. Jager MJ, Shields CL, Cebulla CM. Uveal melanoma. Nat Rev Dis Primers 2020; 6: 24.

25. Kaliki S, Shields CL, Shields JA. Uveal melanoma: estimating prognosis. Indian J Ophthalmol 2015; 63: 93-102.

26. Kuzmanović Elabjer B, Bušić M, Bjeloš M. A non-contiguous recurrence of the ciliary body melanoma - is cataract surgery a risk factor? Int J Ophthalmol 2020; 13: 681-683.

27. Shields CL, Furuta M, Thangappan A. Metastasis of uveal melanoma millimeter-by-millimeter in 8033 consecutive eyes. Arch Ophthalmol 2009; 127: 989-998.

28. Messer JA, Zuhour RJ, Haque W et al. Eye plaque brachytherapy versus enucleation for ocular melanoma: an analysis from the National Cancer Database. J Contemp Brachytherapy 2020; 12: 303-310. 